Research Paper

\title{
Current Smoking has a Detrimental Effect on Survival for Epidermal Growth Factor Receptor (EGFR) and Anaplastic Lymphoma Kinase (ALK) negative Advanced non-squamous Non-small Cell Lung Cancer (NSCLC) Patients Treated with Pemetrexed Continuation Maintenance
}

Liping Lin ${ }^{1} 2^{*}$, Juanjuan Zhao ${ }^{3 *}$, Jiazhu Hu¹,2, Guorong Zou ${ }^{1,2}$, Fuxi Huang1, 2, Jianjun Han ${ }^{1,2}$, Yan He1, , Xiaolong $\mathrm{Cao}^{1,2}$

1. Department of Oncology, Panyu Central Hospital, Guangzhou, China, 511400

2. Cancer Institute of Panyu, Guangzhou, China, 511400

3. School of Nursing, Sun Yat-sen University, Guangzhou, China, 510000

* These authors contributed equally to this work.

$\square$ Corresponding author: Xiaolong Cao, E-mail: pyzh1616@sina.com. Address: Department of Oncology, Panyu Central Hospital, No.8 Fuyu Road East, Panyu, Guangzhou, Guangdong Province, People's Republic of China. Fax: +86(020)34858309; Tel: +86(020)34859212

(c) Ivyspring International Publisher. This is an open access article distributed under the terms of the Creative Commons Attribution (CC BY-NC) license (https://creativecommons.org/licenses/by-nc/4.0/). See http://ivyspring.com/terms for full terms and conditions.

Received: 2018.01.10; Accepted: 2018.04.13; Published: 2018.05.25

\begin{abstract}
Objectives: The aim of this study is to investigate the predictive value of smoking history on treatment outcomes of pemetrexed continuation maintenance therapy in advanced non-squamous NSCLC patients without EGFR mutations and ALK rearrangements.

Methods: 71 consecutive EGFR and ALK negative advanced non-squamous NSCLC patients who had received pemetrexed continuation maintenance therapy at least two cycles were retrospectively analyzed in our single center. The enrolled patients were categorized into two groups as never-/former light smokers and current smokers according to their smoking history.

Results: In the 71 non-squamous NSCLC patients, 30 (42.3\%) were never-/former light smokers and 41 (57.7\%) were current smokers. The objective response rate (ORR) of never-/former light smokers was significantly higher than that of current smokers $(26.7 \%$ vs. $7.3 \%, p=0.026)$. Never-/former light smokers showed significantly longer progression free survival (PFS) $(6.6[95 \% \mathrm{Cl} 5.3-7.9]$ months vs. 5.1 [95\% Cl 3.5-6.7] months; HR: $0.557,95 \% \mathrm{Cl} 0.339-0.915, \mathrm{p}=0.021)$ and overall survival (OS) $(17.3[95 \% \mathrm{Cl}$ 14.4-20.2] months vs. 15.7 [95\% Cl 12.0-19.4] months; HR: $0.578,95 \% \mathrm{Cl} 0.338-0.986, \mathrm{p}=0.044$ ) than current smokers. Multivariate analysis identified smoking history was an independent predictive factor for PFS and OS.

Conclusions: Current smoking is an independent negative predictive factor of outcomes for pemetrexed continuation maintenance therapy in advanced non-squamous NSCLC patients without EGFR mutations and ALK rearrangements.
\end{abstract}

Key words: Smoking history, Predictor, Pemetrexed, Maintenance therapy, Non-small cell lung cancer

\section{Introduction}

Non-small cell lung cancer (NSCLC) is still the main cause of cancer-related deaths in the world [1].
At the time of diagnosis, about $60 \%-70 \%$ of NSCLC patients are locally advanced or metastatic disease [2]. 
Platinum-based combinations as first-line treatment followed by switch or continuation maintenance therapy is recommended by guidelines in driver gene negative patients, especially first-line pemetrexedplatinum chemotherapy followed by pemetrexed continuation maintenance therapy for advanced non-squamous NSCLC patients, resulting in significant prolongation of PFS and OS over placebo as the results of PARAMOUNT study [3-5]. However, some patients do poorly treating with pemetrexed continuation maintenance, whereas others can have prolonged lives despite advanced disease. This suggests clinical heterogeneity among patients treated with pemetrexed continuation maintenance therapy, but mechanisms and clinical factors showing different efficacies are still largely unknown.

Results of several studies have indicated that low mRNA level or negative protein expression of thymidylate synthase (TS) were favorable predictors of response rate and survival in NSCLC treated with pemetrexed-based chemotherapy [6-11]. However, widely testing TS status has not been routinely applied in clinical practice due to lack of sufficient biopsy specimens, high cost-effectiveness, limited testing technology, and so on. Therefore, it is necessary to obtain some useful clinical factors to predict the efficacy of pemetrexed-based chemotherapy for non-squamous NSCLC patients especially those without EGFR mutations and ALK rearrangements. As we know, smoking history is an useful prognostic factor for survival outcome in NSCLC $[12,13]$, but the predictive value of smoking history on pemetrexed continuation maintenance therapy for EGFR and ALK negative advanced non-squamous NSCLC patients yet to be determined.

Accordingly, to investigate whether smoking history can predict the efficacies of pemetrexed continuation maintenance therapy, we consecutively analyzed outcomes of EGFR and ALK negative non-squamous NSCLC patients to pemetrexed continuation maintenance therapy according to their smoking history in our single institution.

\section{Materials and methods}

\section{Study population}

A cohort of 71 consecutive advanced non-squamous NSCLC patients without EGFR mutations and ALK rearrangements who received pemetrexed continuation maintenance therapy at Guangzhou Panyu Central Hospital between September 2011 and November 2015 were enrolled in this retrospective study. Patients who met the criteria as follows were registered: cytologically or histologically confirmed non-squamous NSCLC, disease stage IIIB or IV according to $7^{\text {th }}$ edition tumor/node/metastasis (TNM) system not amenable to curative therapy, without EGFR mutations and ALK rearrangements, has at least one measurable target lesion, Eastern Cooperative Oncology Group Performance Status (ECOG PS) of 0-2, treated with 4-6 cycles pemetrexed-platinum chemotherapy with disease control in first-line, with pemetrexed continuation maintenance therapy at least 2 cycles. According to their smoking history, the enrolled patients were categorized into two groups as never(smoked <100 cigarettes in their life-time)/former light smokers (stopped smoking at least 15 years ago, with a total of $\leq 10$ pack-years of smoking) and current smokers (do not meet standards of never smokers or former light smokers). This study was approved by the institutional ethical committee of our hospital.

\section{Treatment schedule}

Patients with disease control after treated with 4-6 cycles of first-line pemetrexed-platinum chemotherapy received at least 2 cycles pemetrexed (500 mg / m2 intravenous infusion 10 minute on days 1 , every 3 weeks) continuation maintenance therapy. Patients were instructed to inject vitamin $B_{12}(1 \mathrm{mg}$ by intramuscular injection every 9 weeks), and took dexamethasone (4 mg orally, twice daily, on days $0-2$ ) and folic acid ( $0.4 \mathrm{mg} /$ day orally, beginning 5-7 days before the first pemetrexed administration and continuing until 3 weeks after the last pemetrexed administration). Pemetrexed continuation maintenance therapy was continued until disease progression, unacceptable adverse events, or decision of the patient or physician.

\section{Analysis of treatment response}

Physical examination, chest radiography, computed tomography (CT) scan or /and magnetic resonance image (MRI) were performed every two cycles to evaluate target and non-target lesions for all patients in pemetrexed continuation maintenance therapy, then the follow-up time was every 2 months after the treatment cessation. Tumor responses were classified as complete response (CR), partial response $(\mathrm{PR})$, stable disease (SD) and progression disease (PD) according to the Response Evaluation Criteria in Solid Tumors (version 1.1) [14].

\section{Statistical analysis}

Pearson's chi-square test or Fisher's exact test were used to analyze categorical variables, and Student t-test or Mann-Whitney test for continuous variables. Median follow-up was calculated using reverse Kaplan-Meier. PFS was measured from the start of pemetrexed continuation maintenance 
therapy to the time of treatment failure (disease progression or death from any cause) or the last follow-up. OS was measured from the start of pemetrexed continuation maintenance therapy to the time of death from any cause or the last visit before November 30, 2017, censored at the date of last follow up. The survival results were analyzed using Cox proportional hazards regression model and presented as Kaplan-Meier estimates with hazard ratio (HR) and 95\% confidence interval (CI). Cox multivariate regression analyses were performed on PFS and OS, and covariates included histology (non-adenocarcinoma vs. adenocarcinoma), age ( $\geq 60$ or < 60 years), extent of metastasis (M1b vs. M1a), gender (male vs. female), smoking history (never-/former light smoker vs. current smoker), performance status (2 vs. 0-1), cycles of first-line chemotherapy (5-6 vs. 4), and response of first-line chemotherapy (PR vs. SD). Statistical significance was set at 5\%. All tests were two-sided and analyses were carried out with SPSS software, version 17.0 (SPSS Inc., Chicago, IL, USA).

\section{Results}

\section{Patient Characteristics}

A total of 71 advanced non-squamous NSCLC patients without EGFR mutations and ALK rearrangements were identified. The median age was 60 (range: 29-78) years in the entire population. All patients were stage IV disease and $88.7 \%$ of patients were PS $0-1$. Of these patients, $30(42.3 \%)$ were never-/former light smokers and 41 (57.7\%) were current smokers. The majority of never-/former light smokers were female $(80.0 \%)$, whereas most of current smokers were male $(95.1 \%)(p<0.001)$. The other clinical characteristics were not significantly different between the two groups. The clinical characteristics of patients categorized by smoking history are summarized in table 1 .

\section{Clinical responses and survival outcomes of pemetrexed continuation maintenance therapy according to smoking history}

The differences of clinical responses of patients to pemetrexed continuation maintenance therapy between never-/former light smokers and current smokers were analyzed. All patients received at least two cycles of pemetrexed continuation maintenance therapy before treatment cessation. The median pemetrexed continuation maintenance cycles were 8 (range: 2 - 20) and 6 (range: 2-16) in never-/former light smokers and current smokers, respectively. The objective response rate (ORR) of pemetrexed continuation maintenance therapy in never-/former light smokers was significantly higher than that in current smokers $(26.7 \%$ vs. $7.3 \%, p=0.026)$. The clinical responses are shown in table 2.

Table 1. Patients' clinical characteristics according to smoking history

\begin{tabular}{|c|c|c|c|c|}
\hline Characteristics & $\begin{array}{l}\text { Total } \\
(\mathrm{n}=71)(\%)\end{array}$ & $\begin{array}{l}\text { Never-/ former light } \\
\text { smokers }(n=30)(\%)\end{array}$ & $\begin{array}{l}\text { Current } \\
\text { smokers } \\
(n=41)(\%)\end{array}$ & $\mathrm{P}$-value \\
\hline Median age (range), years & $60(29-78)$ & $58.5(35-76)$ & $60(29-78)$ & 0.228 \\
\hline \multicolumn{5}{|l|}{ Age, years } \\
\hline$<60$ & $33(46.5)$ & $16(53.5)$ & $17(41.5)$ & \multirow[t]{2}{*}{0.322} \\
\hline$\geq 60$ & $38(53.5)$ & $14(46.7)$ & $24(58.5)$ & \\
\hline \multicolumn{5}{|l|}{ Gender } \\
\hline Male & $45(63.4)$ & $6(20.0)$ & $39(95.1)$ & \multirow[t]{2}{*}{$<0.001$} \\
\hline Female & $26(36.6)$ & $24(80.0)$ & $2(4.9)$ & \\
\hline \multicolumn{5}{|l|}{ Performance status } \\
\hline $0-1$ & $63(88.7)$ & $26(86.7)$ & $37(90.2)$ & \multirow[t]{2}{*}{0.714} \\
\hline 2 & $8(11.3)$ & $4(13.3)$ & $4(9.8)$ & \\
\hline \multicolumn{5}{|l|}{ Histology } \\
\hline Adenocarcinoma & $65(91.5)$ & $27(90.0)$ & $38(92.7)$ & \multirow[t]{3}{*}{0.795} \\
\hline Large cell carcinoma & $1(1.4)$ & $0(0.00)$ & $1(2.4)$ & \\
\hline $\begin{array}{l}\text { Not otherwise specified, } \\
\text { NOS }\end{array}$ & $5(7.0)$ & $3(10.0)$ & $2(4.9)$ & \\
\hline \multicolumn{5}{|l|}{ Extent of metastasis } \\
\hline M1a & $25(35.2)$ & $10(33.3)$ & $15(36.6)$ & \multirow[t]{2}{*}{0.777} \\
\hline M1b & $46(64.8)$ & $20(66.7)$ & $26(63.4)$ & \\
\hline \multicolumn{5}{|l|}{$\begin{array}{l}\text { First-line chemotherapy } \\
\text { regimens }\end{array}$} \\
\hline Pemetrexed-cisplatin & $57(80.3)$ & $23(76.7)$ & $34(82.9)$ & \multirow[t]{2}{*}{0.513} \\
\hline Pemetrexed-carboplatin & $14(19.7)$ & $7(23.3)$ & $7(17.1)$ & \\
\hline \multicolumn{5}{|l|}{$\begin{array}{l}\text { Cycles of first-line } \\
\text { chemotherapy }\end{array}$} \\
\hline four & $52(73.2)$ & $23(76.7)$ & $29(70.7)$ & \multirow[t]{2}{*}{0.577} \\
\hline five-six & $19(26.8)$ & $7(23.3)$ & $12(29.3)$ & \\
\hline \multicolumn{5}{|l|}{$\begin{array}{l}\text { Response of first-line } \\
\text { chemotherapy }\end{array}$} \\
\hline Partial reponse (PR) & $38(53.5)$ & $20(66.7)$ & $18(43.9)$ & \multirow[t]{2}{*}{0.057} \\
\hline Stable disease (SD) & $33(46.5)$ & $10(33.3)$ & $23(56.1)$ & \\
\hline \multicolumn{5}{|l|}{ Second-line treatment } \\
\hline Yes & $53(74.6)$ & $24(80.0)$ & $29(70.7)$ & \multirow[t]{2}{*}{0.375} \\
\hline No & $18(25.4)$ & $6(20.0)$ & $12(29.3)$ & \\
\hline
\end{tabular}

Table 2. Response according to smoking history

\begin{tabular}{lllll}
\hline Response & $\begin{array}{l}\text { Total } \\
(\mathrm{n}=71)(\%)\end{array}$ & $\begin{array}{l}\text { Never-/former light } \\
\text { smokers }(\mathrm{n}=30)(\%)\end{array}$ & $\begin{array}{l}\text { Current } \\
\text { smokers } \\
(\mathrm{n}=41)(\%)\end{array}$ & P - value \\
\hline Partial response (PR) & $11(15.5 \%)$ & $8(26.7 \%)$ & $3(7.3 \%)$ & \\
$\begin{array}{l}\text { Stable disease (SD) } \\
\text { Progression disease } \\
\text { (PD) }\end{array}$ & $16(62.0 \%)$ & $18(60.0 \%)$ & $26(63.4 \%)$ & $12(29.3 \%)$ \\
$\begin{array}{l}\text { Objective response } \\
\text { rate, \% }\end{array}$ & $15.5 \%$ & $4(13.3 \%)$ & $7.3 \%$ & 0.026 \\
\hline
\end{tabular}

Never-/former light smokers had significantly longer PFS (6.6 [95\% CI 5.3-7.9] months vs. 5.1 [95\% CI 3.5-6.7] months; HR: 0.557, 95\% CI 0.339-0.915, $\mathrm{p}=$ 0.021) (Figure 1a) and overall survival (OS) (17.3 [95\% CI 14.4-20.2] months vs. 15.7 [95\% CI 12.0-19.4] months; HR: 0.578, 95\% CI 0.338-0.986, $\mathrm{p}=0.044$ ) (Figure 2a) than current smokers. Moreover, similar trends on PFS (9.6 [95\% CI 8.3-10.9] months vs. 8.7 [95\% CI 7.4-10.0] months; HR: 0.572, 95\% CI 0.349-0.937, $\mathrm{p}=0.026$ ) (Figure $1 \mathrm{~b})$ and OS (21.5 [95\% CI 16.7-26.3] months vs. 18.5 [95\% CI 14.7-22.3] months; HR: $0.581,95 \%$ CI $0.342-0.989, p=0.045$ ) 
(Figure $2 b$ ) were also found between never-/former light smokers and current smokers if survival time was calculated from the start of first-line pemetrexed-platinum chemotherapy.

\section{Univariate and multivariate analysis of survival outcomes on pemetrexed continuation maintenance therapy}

In univariate analysis, performance status (2 vs. 0-1), smoking history (never-/ former light smoker vs. current smoker) and extent of metastasis (M1b vs. M1a) were found to be significantly associated with
PFS of pemetrexed continuation maintenance therapy (Table 3). Subsequently, the multivariate analysis identified smoking history (HR: 0.387, 95\% CI $0.182-0.822, \mathrm{p}=0.014)$ as independent predictor for PFS (Table 3). However, only smoking history affected OS regardless of univariate or multivariate analysis (HR: 0.312, 95\% CI 0.134-0.724, p = 0.007) (Table 4). Taken together, current smoking independently predicts poorer PFS and OS for EGFR and ALK negative non-squamous NSCLC patients treated with pemetrexed continuation maintenance.
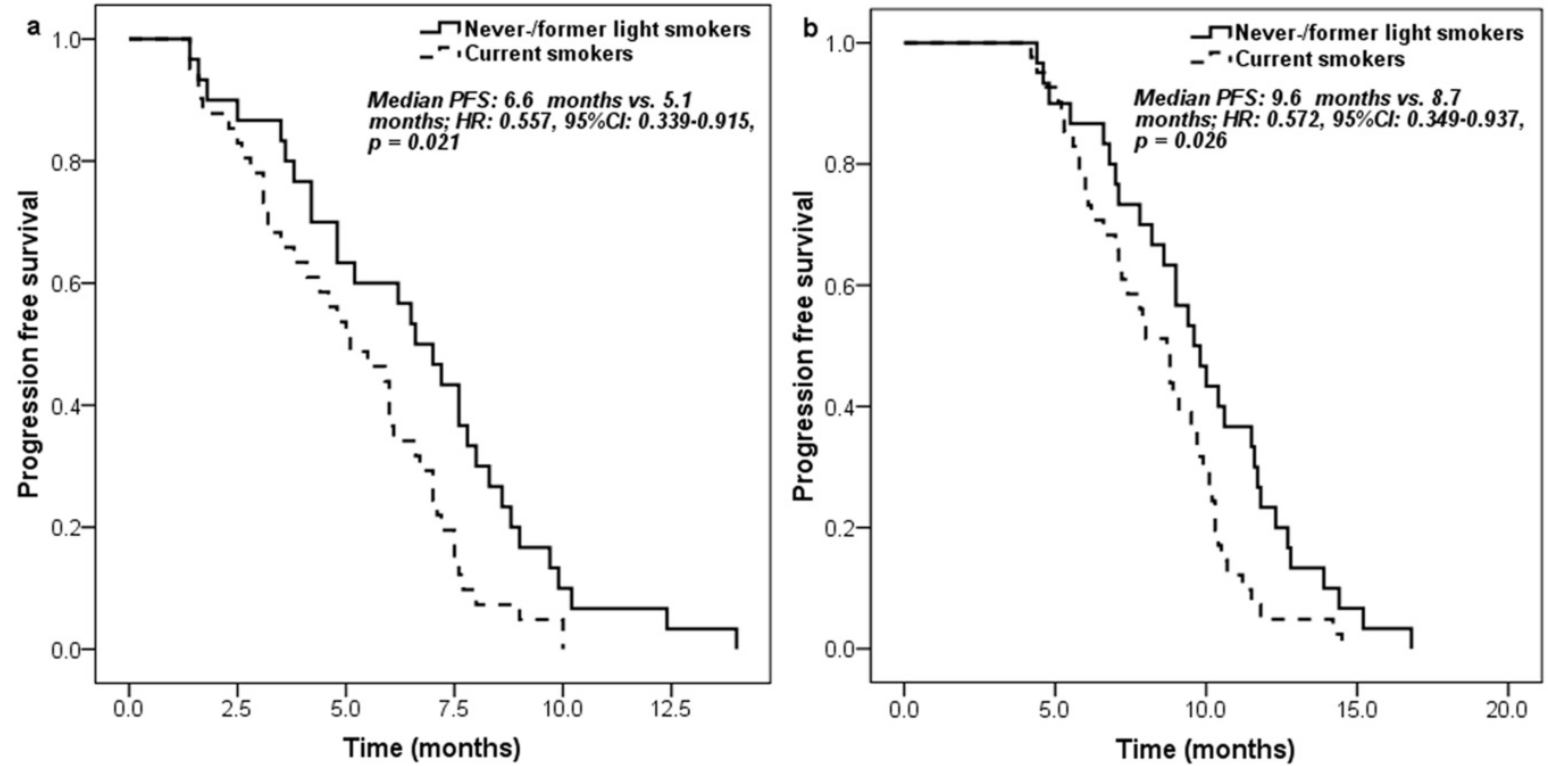

Figure 1. Kaplan-Meier curve for progression free survival according to the smoking history of patients: a. measured from pemetrexed continuation maintenance therapy; $b$. measured from pemetrexed-platinum first-line chemotherapy.
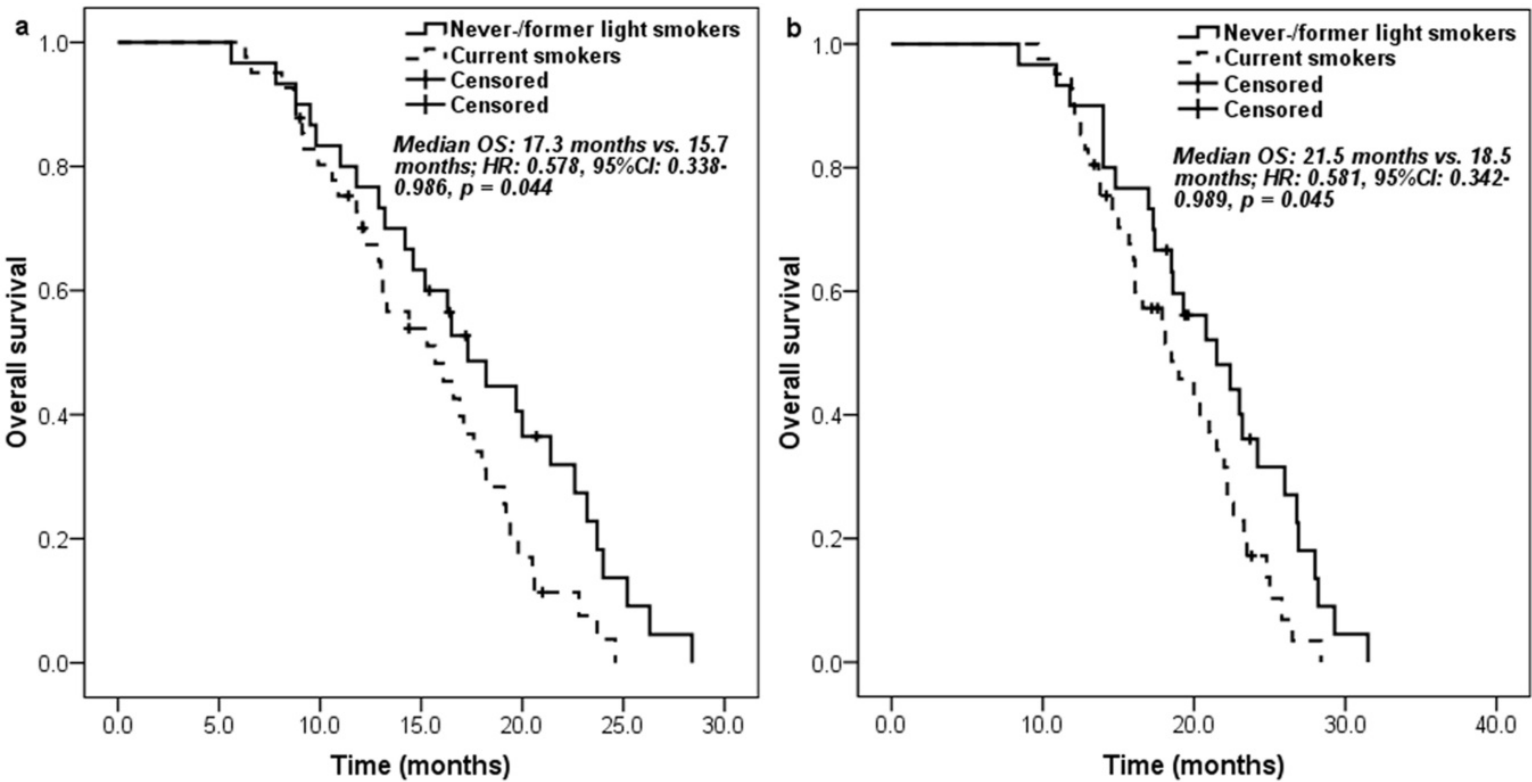

Figure 2. Kaplan-Meier curve for overall survival according to the smoking history of patients: a. measured from pemetrexed continuation maintenance therapy; b. measured from pemetrexed-platinum first-line chemotherapy. 
Table 3. Predictors of PFS (measured from the start of pemetrexed maintenance therapy) analyzed by the Cox regression model

\begin{tabular}{|c|c|c|c|c|}
\hline \multirow[t]{2}{*}{ Variables } & \multicolumn{2}{|l|}{ Univariate analysis } & \multicolumn{2}{|l|}{ Mutivariate analysis } \\
\hline & HR (95\% CI) & $\mathrm{P}$ - value & HR $(95 \% \mathrm{CI})$ & $\mathrm{P}$ - value \\
\hline Smoking history (never-/ former light smoking vs. current smoking) & $0.557(0.339-0.915)$ & 0.021 & $0.387(0.182-0.822)$ & 0.014 \\
\hline Age ( $\geq 60$ vs. $<60$ years) & $1.130(0.703-1.815)$ & 0.614 & $1.142(0.692-1.884)$ & 0.604 \\
\hline Gender (male vs. female) & $1.374(0.834-2.263)$ & 0.213 & $0.786(0.363-1.701)$ & 0.541 \\
\hline Histology (non-adenocarcinoma vs. adenocarcinoma) & $1.111(0.472-2.617)$ & 0.810 & $1.479(0.525-4.166)$ & 0.459 \\
\hline Extent of metastasis (M1b vs. M1a) & $1.725(1.045-2.848)$ & 0.033 & $1.681(1.000-2.827)$ & 0.050 \\
\hline Performance status (2 vs. $0-1$ ) & $2.327(1.056-5.130)$ & 0.036 & $2.061(0.924-4.597)$ & 0.077 \\
\hline Cycles of first-line chemotherapy (5-6 vs. 4 ) & $0.684(0.400-1.171)$ & 0.166 & $0.605(0.333-1.099)$ & 0.099 \\
\hline Response of first-line chemotherapy (PR vs. SD) & $1.190(0.742-1.908)$ & 0.470 & $1.603(0.933-2.754)$ & 0.087 \\
\hline
\end{tabular}

Table 4. Predictors of OS (measured from the start of pemetrexed maintenance therapy) analyzed by the Cox regression model

\begin{tabular}{|c|c|c|c|c|}
\hline \multirow[t]{2}{*}{ Variable } & \multicolumn{2}{|l|}{ Univariate analysis } & \multicolumn{2}{|l|}{ Mutivariate analysis } \\
\hline & $\mathrm{HR}(95 \% \mathrm{CI})$ & $P$ - value & $\operatorname{HR}(95 \% \mathrm{CI})$ & $\mathrm{P}$ - value \\
\hline Smoking history (never-/ former light smoking vs. current smoking) & $0.578(0.338-0.986)$ & 0.044 & $0.312(0.134-0.724)$ & 0.007 \\
\hline Age ( $\geq 60$ vs. $<60$ years) & $0.922(0.554-1.533)$ & 0.754 & $1.142(0.665-1.959)$ & 0.630 \\
\hline Gender (male vs. female) & $1.241(0.718-2.143)$ & 0.440 & $0.488(0.208-1.144)$ & 0.099 \\
\hline Histology (non-adenocarcinoma vs. adenocarcinoma) & $2.352(0.819-6.755)$ & 0.112 & $3.128(0.917-10.669)$ & 0.069 \\
\hline Extent of metastasis (M1b vs. M1a) & $1.167(0.688-1.981)$ & 0.567 & $1.069(0.619-1.847)$ & 0.682 \\
\hline Performance status ( 2 vs. $0-1$ ) & $2.002(0.822-4.874)$ & 0.126 & $2.002(0.804-4.986)$ & 0.136 \\
\hline Cycles of first-line chemotherapy (5-6 vs. 4) & $1.189(0.714-1.978)$ & 0.506 & $0.972(0.517-1.830)$ & 0.931 \\
\hline Response of first-line chemotherapy (PR vs. SD) & $1.188(0.667-2.115)$ & 0.558 & $1.743(0.967-3.144)$ & 0.065 \\
\hline
\end{tabular}

\section{Discussion}

To the best of our knowledge, this is the first study evaluating the effect of smoking history on survival for EGFR and ALK negative advanced non-squamous NSCLC patients treated with pemetrexed continuation maintenance. In our study, compared to the current smokers, never-/former light smoking non-squamous NSCLC patients had significantly higher ORR to pemetrexed continuation maintenance therapy. Additionally, significantly longer PFS and OS were observed in never-/former light smoking non-squamous NSCLC patients regardless of the survival time was calculated from the start of first-line or maintenance therapy. After adjusting for the other clinicopathologic variables, smoking history was identified as an independent predictive factor for PFS and OS. Collectively, our study showed current smoking had a detrimental impact on PFS and OS for EGFR and ALK negative advanced non-squamous NSCLC patients treated with pemetrexed continuation maintenance, suggesting early emergence of pemetrexed resistance and the necessity for more effective maintenance therapy regimens for current smokers.

Park et al. investigated the predictive factors for long-term response duration in non-squamous cell lung cancer patients treated with pemetrexed-based chemotherapy, and the multivariate analysis results indicated never-smoking status was associated with better survival outcome to pemetrexed-based therapy [15]. However, there were many patients with EGFR mutations and ALK rearrangements in the study, and it also mixed the treatment results of different lines. Although a report conducted by Igawa with colleagues for wild-type non-squamous NSCLC patients indicated disease control rate and PFS of pemetrexed monotherapy differed significantly between current smokers and never-smokers/former light smokers [16], the patients were not treated with the same line of pemetrexed. In general, large heterogeneity was existed in enrolled patients and treatment lines of pemetrexed in these studies. However, the present study was different with aforementioned studies, as it was conducted in non-squamous population without EGFR mutations and ALK rearrangements. In addition, all patients were received the same pemetrexed treatment schedule in our study. Therefore, this study has less confounding factors and the results are reproducible. Furthermore, even though survival time was calculated from the start of first-line pemetrexed-platinum chemotherapy, never-/former light smokers still had significantly longer PFS and OS, which was consistent with the findings of Shaw's that PFS of first-line platinum/pemetrexed may be prolonged in never/light-smoking patients [17]. But it was notably that PS was not an independent predictor of PFS in multivariate analysis in our data, which was different with the result of Igawa's [16], and this may be due to the small sample size.

Smoking history predicting outcomes of pemetrexed continuation maintenance therapy is valuable in determining the optimal maintenance therapy strategy for an individual patient, and potential molecular mechanisms may also exist. As we know, there are more smokers in squamous lung cancer compared with non-squamous lung cancer [18]. Coincidentally, TS expression is also higher in squamous lung cancer patients than non-squamous 
lung cancer patients $[19,20]$. These results indicate TS expression may be closely associated with smoking history. Studies had indeed demonstrated that TS expression was significantly lower in never-smokers than that in smokers in non-squamous NSCLC patients [21, 22], whereas it is well known that low TS expression suggests better survival outcomes in non-squamous NSCLC patients treated with pemetrexed-based regimens [6-11]. Moreover, a genomic analysis shown that smoking lung cancer patients have much higher genetic changes than that of never smokers [23], which strongly indicates more heterogeneous tumor behavior and chemo-resistance in smokers. Therefore, we speculated that higher expression of TS and drug resistance genes in current smokers than never-/former light smokers may be the main molecular mechanisms, which result in smoking non-squamous NSCLC patients having earlier drug-resistance to pemetrexed continuation maintenance therapy. These findings imply that special management strategy should be employed or explored for current smoking non-squamous NSCLC patients in maintenance therapy. However, a previous study did not find significant differences in the efficacy of pemetrexed-cisplatin versus gemcitabinecisplatin in first-line treatment for current smokers [24]. Also, no prior study revealed that tumor response was significantly different among the other drugs and pemetrexed for current smoking non-squamous NSCLC patients in maintenance therapy. Thus, developing new drugs for current smokers based on a more in-depth research on tumor genetic characteristics of these patients may be the way to solve this issue.

However, some limitations in the present study should be mentioned. First, our study was performed retrospectively in single institution with small sample size and the patients were not treated according to an established protocol, which means a selection bias may be exist. Second, smokers receiving some toxicity drugs demonstrated increased clearance and lower systemic exposure, which may result in suboptimal therapy and poor outcomes [25]. But no pharmacokinetic data could be used in our study to validate the different efficacy of pemetrexed continuation maintenance therapy between never-/former light smokers and current smokers. Lastly, a previous study revealed that low TS expression was associated with improved PFS and OS in non-squamous NSCLC patients treated with pemetrexed continuation maintenance therapy [26], however, we couldn't analyze the correlation of TS expression with survival because of insufficient tumor tissues.

\section{Conclusions}

In conclusion, this study demonstrated a detrimental effect of current smoking on survival outcomes in EGFR and ALK negative non-squamous NSCLC patients treated with pemetrexed continuation maintenance. Although current smokers had distinctly poorer PFS and OS on pemetrexed continuation maintenance therapy, it may be still reasonable to select maintenance therapy in these patients. We suggest that new clinical trials should be carried out in the future, to compare pemetrexed with other drugs in maintenance therapy for current smokers to identify the optimal maintenance regimens. Taken together, our findings revealed smoking history can be considered as an essential factor in clinical practice regarding pemetrexed continuation maintenance therapy in wild-type non-squamous NSCLC patients, and the patients' smoking history should be detailed collected before the initiation of pemetrexed continuation maintenance.

\section{Acknowledgements}

This work was supported by Medical Science and Technology Research Foundation of Guangdong Province (Grant No. A2016307).

\section{Competing Interests}

The authors have declared that no competing interest exists.

\section{References}

1. Siegel RL, Miller KD, Jemal A. Cancer statistics, 2017. CA Cancer J Clin. 2017; 67(1): 7-30.

2. Mountain CF. Revisions in the International System for Staging Lung Cancer. Chest. 1997; 111 (6): 1710-7.

3. [Internet] NCCN Clinical Practice Guidelines in Oncology: Non-Small Cell Lung Cancer, Version 1. 2018. https://www.nccn.org/professionals/ physician_gls/pdf/ nscl.pdf.

4. Pazares L, De M F, Dediu M, et al. Maintenance therapy with pemetrexed plus best supportive care versus placebo plus best supportive care after induction therapy with pemetrexed plus cisplatin for advanced non-squamous non-small-cell lung cancer (PARAMOUNT): a double-blind, phase 3, randomised controlled trial. Lancet Oncol. 2012, 13(3): 247-55.

5. Paz-Ares LG, de Marinis F, Dediu M, et al. PARAMOUNT: Final overall survival results of the phase III study of maintenance pemetrexed versus placebo immediately after induction treatment with pemetrexed plus cisplatin for advanced nonsquamous non-small-cell lung cancer. J Clin Oncol. 2013, 31(23): 2895-902.

6. Wang L, Wang R, Pan Y, et al. The pemetrexed-containing treatments in the non-small cell lung cancer is -/low thymidylate synthase expression better than + /high thymidylate synthase expression: a meta-analysis. BMC Cancer. 2014; 14: 205

7. Liu Q, Yu Z, Xiang Y, et al. Prognostic and predictive significance of thymidylate synthase protein expression in non-small cell lung cancer: a systematic review and meta-analysis. Cancer Biomark. 2015; 15: 65-78.

8. Arevalo E, Castanon E, Lopez I, et al. Thymidylate synthase polymorphisms in genomic DNA as clinical outcome predictors in a European population of advanced non-small cell lung cancer patients receiving pemetrexed. J Transl Med 2014; 12: 98

9. Sun JM, Ahn JS, Jung SH, et al. Pemetrexed Plus Cisplatin Versus Gemcitabine Plus Cisplatin According to Thymidylate Synthase Expression in Nonsquamous Non-Small-Cell Lung Cancer: A Biomarker-Stratified Randomized Phase II Trial. J Clin Oncol. 2015, 33(22):2450-6.

10. Lin CS, Liu TC, Lai JC, et al. Evaluating the Prognostic Value of ERCC1 and Thymidylate Synthase Expression and the Epidermal Growth Factor 
Receptor Mutation Status in Adenocarcinoma Non-Small-Cell Lung Cancer. Int J Med Sci. 2017, 14(13):1410-1417.

11. Cardona AF, Rojas L, Wills B, et al. Pemetrexed/Carboplatin/Bevacizumab followed by Maintenance Pemetrexed/Bevacizumab in Hispanic Patients with Non-Squamous Non-Small Cell Lung Cancer: Outcomes according to Thymidylate Synthase Expression. PLoS One. 2016, 11(5):e0154293.

12. Maeda R, Yoshida J, Ishii G, et al. The prognostic impact of cigarette smoking on patients with non-small cell lung cancer. J Thorac Oncol. 2011, 6(4):735-42.

13. Ferketich AK, Niland JC, Mamet R, et al. Smoking status and survival in the national comprehensive cancer network non-small cell lung cancer cohort. Cancer. 2013, 119(4):847-53.

14. Eisenhauer EA, Therasse P, Bogaerts J, et al. New response evaluation criteria in solid tumours: revised RECIST guideline (version 1.1). Eur J Cancer. 2009; 45(2): 228-47.

15. Park S, Kim HJ, Choi CM, et al. Predictive factors for a long-term response duration in non-squamous cell lung cancer patients treated with pemetrexed. BMC Cancer. 2016, 16:417.

16. Igawa S, Sasaki J, Otani S, et al. Smoking History as a Predictor of Pemetrexed Monotherapy in Patients with Non-Squamous Non-Small Cell Lung Cancer. Oncology. 2016, 91(1):41-7.

17. Shaw AT, Varghese AM, Solomon BJ, et al. Pemetrexed-based chemotherapy in patients with advanced, ALK-positive non-small cell lung cancer. Ann Oncol. 2013, 24(1):59-66.

18. Khuder SA. Effect of cigarette smoking on major histological types of lung cancer: a meta-analysis. Lung Cancer. 2001, 31(2-3):139-48.

19. Maus MK, Mack PC, Astrow SH, et al. Histology-related associations of ERCC1, RRM1, and TS biomarkers in patients with non-small-cell lung cancer: implications for therapy. J Thorac Oncol. 2013, 8(5):582-6.

20. Wynes MW, Konopa K, Singh S, et al. Thymidylate synthase protein expression by IHC and gene copy number by SISH correlate and show great variability in non-small cell lung cancer. J Thorac Oncol. 2012, 7(6):982-92.

21. Huang $\mathrm{Y}$, Guo $\mathrm{X}$, Wang $\mathrm{H}$, et al. Predictive value of thymidylate synthase for the prognosis and survival of lung adenocarcinoma patients. Oncol Lett. 2015, 9(1):252-256.

22. Sun JM, Han J, Ahn JS, et al. Significance of thymidylate synthase and thyroid transcription factor 1 expression in patients with nonsquamous non-small cell lung cancer treated with pemetrexed-based chemotherapy. J Thorac Oncol. 2011, 6(8):1392-9.

23. Govindan R, Ding L, Griffith M, et al. Genomic landscape of non-small cell lung cancer in smokers and never-smokers. Cell. 2012, 150(6):1121-34.

24. Scagliotti GV, Parikh P, von Pawel J, et al. Phase III study comparing cisplatin plus gemcitabine with cisplatin plus pemetrexed in chemotherapy-naive patients with advanced-stage non-small-cell lung cancer. J Clin Oncol. 2008, 26(21):3543-51.

25. O'Malley M, King AN, Conte M, et al. Effects of cigarette smoking on metabolism and effectiveness of systemic therapy for lung cancer. J Thorac Oncol. 2014, 9(7):917-26.

26. Yang M, Fan WF, Pu XL, et al. The role of thymidylate synthase in non-small cell lung cancer treated with pemetrexed continuation maintenance therapy. J Chemother. 2017, 29(2):106-112. 